in vivo $34: 3349-3360(2020)$

doi:10.21873/invivo.12173

\title{
The Systemic Immune-Inflammation Index Predicts Clinical Outcomes in Kidney Transplant Recipients
}

\author{
SAMANTHA E. HALPERN ${ }^{1}$, DIMITRIOS MORIS ${ }^{2}$, BRIAN I. SHAW ${ }^{2}$, \\ MADISON K. KRISCHAK ${ }^{1}$, DANAE G. OLASO ${ }^{1}$, SAMUEL J. KESSELI ${ }^{2}$, \\ KADIYALA RAVINDRA ${ }^{2}$, LISA M. MCELROY ${ }^{2}$ and ANDREW S. BARBAS ${ }^{2}$ \\ ${ }^{1}$ School of Medicine, Duke University, Durham, NC, U.S.A.; \\ ${ }^{2}$ Department of Surgery, Duke University Medical Center, Durham, NC, U.S.A.
}

\begin{abstract}
Background: Outcomes after kidney transplantation (KTx) remain limited by delayed graft function (DGF) and acute rejection. Non-invasive biomarkers may help identify patients at increased risk for these events. We examined the association between the systemic immune-inflammation index (SII), a novel inflammatory biomarker, and outcomes after KTx and evaluated its ability to predict post-transplant prognosis. Patients and Methods: Adult patients who underwent primary KTx at our institution between 2016-2019 were included. SII was calculated from pre-transplant complete blood counts as the ratio of the neutrophil count to the lymphocyte count multiplied by the platelet count. The cutoff between high and low SII was determined by maximizing the area under the curve. Multivariable logistic and Cox regression were used to identify factors associated with DGF and patient, rejectionfree, and graft survival respectively. Results: Overall, 378 KTx recipients were included; 224 (59.3\%) had high SII. On unadjusted analysis, high SII was associated with reduced odds of DGF, and improved patient and rejection-free survival. After adjustment, high SII was independently associated with improved patient survival alone. Multivariable models incorporating SII performed well for the prediction of DGF (cstatistic $=0.755)$ and patient survival $(c$-statistic $=0.786)$, though rejection-free survival was more difficult to predict (cstatistic=0.635). Conclusion: SII demonstrated limited utility as an independent predictor of outcomes after KTx. However, in combination with other clinically relevant parameters, SII
\end{abstract}

This article is freely accessible online.

Correspondence to: Dimitrios Moris, MD, MSc, Ph.D., Box 3512, DUMC, Department of Surgery, Duke University Medical Center, Durham, NC 27710, U.S.A. Tel: +1 2165716614, e-mail: dimitrios.moris@duke.edu

Key Words: Systemic immune-inflammation index, kidney transplantation, biomarkers, delayed graft function, acute rejection. is a useful predictor of post-KTx prognosis. Validation of this novel inflammatory biomarker in a multi-institutional study is needed to further elucidate its practical applications in transplantation.

Kidney transplantation is the standard of care for patients with end-stage renal disease (ESRD), offering significant health and quality of life benefits compared to remaining on dialysis (1). Despite a steady increase in the number of kidney transplants performed in the US, delayed graft function (DGF) and acute rejection $(\mathrm{AR})$ remain prevalent, hindering optimization of patient and allograft survival $(2,3)$. In the past decade, research on the transplant community has given increasing attention to the development and identification of novel biomarkers that allow for rapid, safe, non-invasive, and accurate detection of immunological injury to the kidney allograft $(4,5)$. Of particular interest are prognostic and predictive biomarkers that can identify patients at increased risk for allograft injury, including $\mathrm{DGF}$ and $\mathrm{AR}(6,7)$.

The neutrophil-to-lymphocyte ratio (NLR) and platelet-tolymphocyte ratio (PLR) are two biomarkers that provide an indication of inflammatory status and have been shown to be strong predictors of negative prognosis in a variety of conditions including cancer $(8,9)$, cardiovascular disease $(10$, 11), ESRD (12), and, more recently, kidney and liver transplantation (13-18). Despite a growing body of evidence examining the utility of NLR and PLR to predict immunologic injury, the literature remains divided regarding the association between these inflammatory markers and development of DGF and AR. Specifically, while some studies have demonstrated an association between high NLR and PLR and development of DGF and AR $(14,15,18)$, others report the opposite findings $(16,17)$. This lack of consensus suggests a need for ongoing investigation to identify additional biomarkers capable of identifying patients at risk of post-transplant immunologic events.

The systemic immune-inflammation index (SII) was recently introduced as a novel and powerful prognostic 
marker of outcomes in patients with cancer (19-21). Initially developed as a prognostic tool for patients undergoing curative resection for hepatocellular carcinoma (HCC), the SII represents an integrated indicator based on peripheral lymphocyte, neutrophil, and platelet counts (19). To date the SII has not been evaluated in the setting of kidney transplantation and little is known about its prognostic value in the transplant field. A recent study examining the prognostic value of SII among patients undergoing liver transplantation for HCC demonstrated an independent association between high SII and overall survival, as well as superior predictive capacity compared to NLR and PLR in these patients (22), suggesting the potential utility of SII more broadly in transplantation.

In pursuit of a novel, safe, non-invasive, and accurate biomarker of immunologic injury after kidney transplantation, we sought to define the association between SII and outcomes after kidney transplantation with particular attention to DGF. In addition, we investigated whether SII better predicted posttransplant prognosis compared with the traditional inflammatory indices PLR and NLR.

\section{Patients and Methods}

Study population. All patients who underwent kidney transplantation at Duke University Hospital between 2016 and 2019 were identified by chart review. Pediatric (age $<18$ years), multiorgan transplant and repeat kidney transplantation recipients, and patients who did not have a pre-transplant complete blood count $(\mathrm{CBC})$ recorded within 6 months of kidney transplantation were excluded. This study was approved by the Duke University Institutional Review Board (Pro00103325).

Study design. Variables of interest were obtained from both institutional charts and the United Network for Organ Sharing. Recipient and operative characteristics included basic demographics (age, sex, race, ethnicity), preoperative characteristics [history of malignancy, pre-transplant panel reactive antibody (PRA)], indication for transplant, operative data (estimated blood loss, intraoperative transfusion requirements, use of ureteral stents, intraoperative drain placement), and donor variables (donor type, cold and warm ischemia time, use of machine perfusion, kidney donor profile index (KDPI), US Public Health Service increased risk status, extended criteria donor, Epstein-Barr virus and cytomegalovirus serologies, graft laterality, and number of human leukocyte antigen mismatches).

Inflammatory indices were defined as follows:

I. NLR: Absolute neutrophil count divided by absolute lymphocyte count.

II. PLR: Absolute platelet count divided by absolute lymphocyte count.

III. SII: Absolute platelet count multiplied by NLR.

Inflammatory indices of interest were calculated using the most recent pre-transplant $\mathrm{CBC}$ with white blood cell differential, measured within 6 months prior to kidney transplantation. At our institution, patients undergoing deceased donor kidney transplantation (DDKT) have a CBC with differential upon admission, however those undergoing living donor kidney transplantation (LDKT) may have blood drawn at their pre- operative evaluation visit; therefore a CBC cut-off of 6 months pretransplant was chosen to capture LDKT patients whose samples were collected more than 24 hours pre-transplant.

Outcomes. The primary outcome of interest was DGF, defined as the need for postoperative dialysis within 7 days of transplantation. Secondary outcomes included patient survival, rejection-free survival, and graft survival. To allow adjustment for additional donor variables that are known to be associated with development of DGF including extended criteria donor status and KDPI (3), the association between the inflammatory indices and the primary and secondary outcomes were assessed for the cohort of DDKT recipients as well as for the entire patient cohort, which included both DDKT and LDKT recipients. Sub-group analysis of LDKT recipients was not performed due to the limited number of patients in this group.

Statistical analysis. Descriptive statistics are presented as medians and interquartile ranges (IQR) for continuous variables and proportions for categorical variables. Recipient and operative characteristics were compared between DDKT and LDKT recipients using Wilcoxon rank-sum tests for continuous variables and Chisquared and Fisher exact tests for categorical variables.

For the comparison of groups with low versus high SII, PLR, and NLR, cut-off values were selected based on maximization of the area under the curve using univariable logistic regression models with DGF as the outcome $(23,24)$. Selected cut-off values were additionally used to determine the association between SII, PLR, and NLR and the secondary outcomes. The optimal cut-off values for SII, PLR, and NLR were determined separately for DDKT recipients and the unstratified patient cohort.

Logistic regression was employed to examine the association between recipient, operative, and inflammatory characteristics and DGF. Variables included in the final multivariable models were selected using backwards stepwise regression with an AIC criterion. Performance of the final models was assessed using the biascorrected c-statistic determined based on cross-validation with 1,000 bootstrapped resamples.

Patient, rejection-free, and graft survival were estimated in an unadjusted fashion using the Kaplan-Meier method. Log-rank tests were used to compare survival between high and low NLR, PLR, and SII groups. Cox regression was employed to examine the association between recipient, operative, and inflammatory characteristics and each of the secondary outcomes. Model selection and assessment of model performance were conducted as described above.

Sensitivity analyses were performed to evaluate the performance of all multivariable models using the inflammatory indices as continuous variables. A two-sided $p$-value less than 0.05 was considered statistically significant. All analyses were performed using R version 3.6.1 (Vienna, Austria).

\section{Results}

Study population and operative characteristics. A total of 378 primary kidney-only transplant recipients met the inclusion criteria. Of those, 327 (86.5\%) underwent DDKT and $51(13.5 \%)$ underwent LDKT. Recipient characteristics for the entire patient cohort and stratified by donor type are summarized in Table I. Compared to DDKT recipients, 
Table I. Recipient characteristics.

\begin{tabular}{|c|c|c|c|c|}
\hline Characteristic & $\begin{array}{c}\text { All kidney } \\
\text { transplants }(\mathrm{N}=378)\end{array}$ & $\begin{array}{c}\text { DDKT } \\
(\mathrm{N}=327)\end{array}$ & $\begin{array}{l}\text { LDKT } \\
(\mathrm{N}=51)\end{array}$ & $p$-Value \\
\hline Age (years), median (IQR) & $55(45-63)$ & $55(46-63)$ & $54(43-62)$ & 0.28 \\
\hline Gender, n $(\%)$ & & & & 0.64 \\
\hline Female & $167(44.2)$ & $146(44.6)$ & $21(41.2)$ & \\
\hline Male & $211(55.8)$ & $181(55.4)$ & $30(58.8)$ & \\
\hline Race, n (\%) & & & & $<0.01$ \\
\hline White & $140(37.0)$ & $104(31.8)$ & $36(70.6)$ & \\
\hline Black & $213(56.3)$ & $201(61.5)$ & $12(23.5)$ & \\
\hline Asian & $5(1.3)$ & $5(1.5)$ & $0(0)$ & \\
\hline Other & $20(5.3)$ & $17(5.2)$ & $3(5.9)$ & \\
\hline Ethnicity (Hispanic), n (\%) & $11(2.9)$ & $11(3.4)$ & $0(0)$ & 0.37 \\
\hline \multicolumn{5}{|l|}{ PRA at transplant (\%), median (IQR) } \\
\hline Class I & $0(0-10)$ & $0(0-13)$ & $0(0-0)$ & $<0.01$ \\
\hline Class II & $0(0-0)$ & $0(0-0)$ & $0(0-0)$ & 0.29 \\
\hline Etiology of kidney disease, $\mathrm{n}(\%)$ & & & & 0.066 \\
\hline Alport syndrome & $4(1.1)$ & $3(0.9)$ & $1(2.0)$ & \\
\hline Calcineurin inhibitor nephrotoxicity & $9(2.4)$ & $5(1.5)$ & $4(7.8)$ & \\
\hline Chronic glomerulonephritis & $11(2.9)$ & $10(3.1)$ & $1(2.0)$ & \\
\hline Congenital obstructive uropathy & $7(1.9)$ & $5(1.5)$ & $2(3.9)$ & \\
\hline Focal glomerular sclerosis & $34(9.0)$ & $29(8.9)$ & $5(9.8)$ & \\
\hline HIV nephropathy & $4(1.1)$ & $4(1.2)$ & $0(0)$ & \\
\hline Hypertensive nephrosclerosis & $80(21.2)$ & $75(22.9)$ & $5(9.8)$ & \\
\hline IgA nephropathy & $17(4.5)$ & $15(4.6)$ & $2(3.9)$ & \\
\hline Lithium toxicity & $3(0.8)$ & $3(0.9)$ & $0(0)$ & \\
\hline Malignant hypertension & $7(1.9)$ & $7(2.1)$ & $0(0)$ & \\
\hline Polycystic kidney disease & $36(9.5)$ & $30(9.2)$ & $6(11.8)$ & \\
\hline Sickle cell anemia & $4(1.1)$ & $4(1.2)$ & $0(0)$ & \\
\hline Systemic lupus erythematosus & $13(3.4)$ & $13(4.0)$ & $0(0)$ & \\
\hline Type 1 diabetes mellitus & $12(3.2)$ & $8(2.4)$ & $4(7.8)$ & \\
\hline Type 2 diabetes mellitus & $104(27.5)$ & $90(27.5)$ & $14(27.5)$ & \\
\hline Vasculitis & $3(0.8)$ & $3(0.9)$ & $0(0)$ & \\
\hline Other & $30(7.9)$ & $23(7.0)$ & $7(13.7)$ & \\
\hline History of pre-transplant malignancy, n (\%) & $38(10.1)$ & $35(10.7)$ & $3(5.9)$ & 0.45 \\
\hline Breast & $5(1.3)$ & $5(1.5)$ & $0(0)$ & $>0.9$ \\
\hline Colorectal & $3(0.8)$ & $3(0.9)$ & $0(0)$ & $>0.9$ \\
\hline Hematological & $9(2.4)$ & $7(2.1)$ & $2(3.9)$ & 0.35 \\
\hline Lung & $1(0.3)$ & $1(0.3)$ & $0(0)$ & $>0.9$ \\
\hline Prostate & $6(1.6)$ & $6(1.8)$ & $0(0)$ & $>0.9$ \\
\hline Renal cell & $8(2.1)$ & $7(2.1)$ & $1(2.0)$ & $>0.9$ \\
\hline Skin & $10(2.6)$ & $10(3.1)$ & $0(0)$ & 0.37 \\
\hline Thyroid & $2(0.5)$ & $2(0.6)$ & $0(0)$ & $>0.9$ \\
\hline NLR, median (IQR) & $2.67(1.94-3.89)$ & $2.62(1.92-3.78)$ & $3.23(2.31-4.62)$ & 0.039 \\
\hline PLR, median (IQR) & $126(99.7-168)$ & $124(98.3-163)$ & $155(108-218)$ & 0.011 \\
\hline SII, median (IQR) & $555(350-869)$ & $545(343-855)$ & $617(418-941)$ & 0.077 \\
\hline
\end{tabular}

DDKT: deceased donor kidney transplants; LDKT: living donor kidney transplants; IQR: Interquartile range; NLR: neutrophil to lymphocyte ratio; PLR: platelet to lymphocyte ratio; PRA: pre-transplant panel reactive antibody; SII: systemic immune-inflammation index. Statistically significant $p$-values are shown in bold.

LDKT recipients were more likely to be White $(70.6 \% \mathrm{vs}$. $31.8 \%, p<0.01)$, have lower pre-transplant class I PRA [median (IQR) 0 (0-13) vs. $0(0-0), p<0.01$ ], and higher PLR and NLR [median (IQR) PLR: 155 (108-218) vs. 124 (98.3163), $p=0.011$; NLR: 3.23 (2.31-4.62) vs. 2.62 (1.92-3.78), $p=0.04]$. There were no significant differences in age, gender, ethnicity, etiology of kidney disease, history of malignancy, or SII between DDKT and LDKT recipients.
Operative characteristics for the entire patient cohort and stratified by donor type are summarized in Table II. Among DDKT recipients, 237 (72.5\%) received a kidney from a donation after brain death donor and $90(27.5 \%)$ received a kidney from a donation after circulatory death donor. LDKT was associated with shorter ischemic times, lower intraoperative blood loss, fewer human leukocyte antigen mismatches, and use of a left kidney allograft (all $p<0.05)$. 
Table II. Operative characteristics.

\begin{tabular}{|c|c|c|c|c|}
\hline Characteristic & $\begin{array}{c}\text { All kidney } \\
\text { transplants }(\mathrm{N}=378)\end{array}$ & $\begin{array}{c}\text { DDKT } \\
(\mathrm{N}=327)\end{array}$ & $\begin{array}{l}\text { LDKT } \\
(\mathrm{N}=51)\end{array}$ & $p$-Value \\
\hline \multicolumn{5}{|l|}{ Cold ischemic time (minutes), median (IQR) } \\
\hline All kidneys & $1,097(782-1498)$ & $1,192(933-1534)$ & $78(62-119)$ & $<0.01$ \\
\hline Non-pumped kidneys only & $276(81-904)$ & $904(623-1140)$ & $78(62-119)$ & $<0.01$ \\
\hline Warm ischemic time (minutes), median (IQR) & $28(23-33)$ & $29(23-33)$ & $26(23-29)$ & 0.013 \\
\hline Total ischemic time (minutes), median (IQR) & $1,138(810-1521)$ & $1,223(955-1563)$ & $100(88-143)$ & $<0.01$ \\
\hline Estimated blood loss (ml), median (IQR) & $150(100-200)$ & $150(100-200)$ & $100(75-200)$ & 0.038 \\
\hline \multicolumn{5}{|l|}{ Transfusion requirement (units), median (IQR) } \\
\hline RBCs & $0(0-0)$ & $0(0-0)$ & $0(0-0)$ & 0.14 \\
\hline FFP & $0(0-0)$ & $0(0-0)$ & $0(0-0)$ & 0.58 \\
\hline Cryo & $0(0-0)$ & $0(0-0)$ & $0(0-0)$ & - \\
\hline Platelets & $0(0-0)$ & $0(0-0)$ & $0(0-0)$ & - \\
\hline Pump used, n (\%) & $273(72.2)$ & $273(83.5)$ & $0(0)$ & $<0.01$ \\
\hline Donor type, n (\%) & & & & - \\
\hline Living & $51(13.5)$ & $0(0)$ & $51(100)$ & \\
\hline $\mathrm{DBD}$ & $237(62.7)$ & $237(72.5)$ & $0(0)$ & \\
\hline DCD & $90(23.8)$ & $90(27.5)$ & $0(0)$ & \\
\hline PHS increased risk, $\mathrm{n}(\%)^{\mathrm{a}}$ & $109(28.8)$ & $109(33.3)$ & - & - \\
\hline Extended criteria donor, $\mathrm{n}(\%)^{\mathrm{a}}$ & $51(13.5)$ & $51(15.6)$ & - & - \\
\hline \multicolumn{5}{|l|}{ Donor serology, n (\%) } \\
\hline EBV-positive & $359(95.0)$ & $316(96.6)$ & $43(84.3)$ & $<0.01$ \\
\hline CMV-positive & $238(63.0)$ & $209(63.9)$ & $29(56.9)$ & 0.33 \\
\hline KDPI $(\%)$, median (IQR) ${ }^{\mathrm{a}}$ & $56(34-71)$ & $56(34-71)$ & - & - \\
\hline HLA mismatch, median (IQR) & $4(3-5)$ & $4(4-5)$ & $4(3-5)$ & $<0.01$ \\
\hline Graft laterality, n (\%) & & & & $<0.01$ \\
\hline Right & $181(47.9)$ & $180(55.0)$ & $1(2.0)$ & \\
\hline Left & $190(50.3)$ & $140(42.8)$ & $50(98.0)$ & \\
\hline Dual & $7(1.9)$ & $7(2.1)$ & $0(0)$ & \\
\hline Ureteral stent used, n (\%) & $258(68.3)$ & $226(69.1)$ & $32(62.7)$ & 0.36 \\
\hline Drain placed in OR, $\mathrm{n}(\%)$ & $89(23.5)$ & $80(24.5)$ & $9(17.6)$ & 0.29 \\
\hline
\end{tabular}

IQR: Interquartile range; NLR: neutrophil to lymphocyte ratio; PLR: platelet to lymphocyte ratio; RBC: red blood cells; DBD: donors from brain death; DCD: donor from circulatory death; cryo: cryoprecipitate; FFP: fresh frozen plasma; EBV: Epstein-Barr virus; CMV: cytomegalovirus; PHS: public health service; HLA: human leukocyte antigen; KDPI; kidney donor profile index; DDKT: deceased donor kidney transplants; LDKT: living donor kidney transplants; SII: systemic immune-inflammation index. OR: operating room. aRecorded for deceased donors only. Statistically significant $p$-values are shown in bold.

Inflammatory indices. For the entire patient cohort, high SII, PLR, and NLR, were defined as $>475.5,>103.4$, and $>3.3$ respectively. Overall, 224 (59.3\%) patients had high SII, 266 (70.4\%) had high PLR, and 136 (36.0\%) had high NLR. Compared to those with high SII, patients with low SII had significantly lower levels of circulating neutrophils and platelets and higher levels of circulating lymphocytes in pretransplant peripheral blood samples (Figure 1). Levels of circulating lymphocytes were also significantly higher among patients with low PLR and low NLR compared to patients in the respective higher groups.

Association between inflammatory indices and DGF. Overall, DGF occurred in 124 (32.8\%) patients. When stratified by donor type, DGF occurred in 122 (37.3\%) DDKT recipients and two (3.9\%) LDKT recipients. On unadjusted analysis of the entire patient cohort, high SII was associated with significantly reduced odds of DGF [odds ratio $(\mathrm{OR})=0.60$,
$95 \%$ confidence interval $(\mathrm{CI})=0.39-0.92, p=0.02]$. High NLR was also associated with reduced odds of DGF $(\mathrm{OR}=0.60$, 95\% CI=0.37-0.94, $p=0.03$ ), while high PLR was not associated with DGF on unadjusted analysis $(\mathrm{OR}=1.49,95 \%$ $\mathrm{CI}=0.92-2.46, p=0.1)$. When adjusted for age, etiology of kidney disease, cold ischemia time, and donor type (donor brain death, donor circulatory death, living), high SII and high NLR were no longer associated with DGF, whereas high PLR was independently associated with a $92 \%$ increase in the odds of DGF (SII: OR=0.64, 95\% CI=0.39-1.04, $p=0.07$; NLR: $\mathrm{OR}=0.65,95 \% \mathrm{CI}=0.38-1.09, p=0.1$; PLR: $\mathrm{OR}=1.92,95 \% \mathrm{CI}=1.12-3.33, p=0.02)$. After the addition of each inflammatory index to the model, the model including PLR was associated with a c-index of 0.761 (95\% CI=0.6930.792 ), outperforming models including SII (c-index 0.755 , 95\% $\mathrm{CI}=0.704-0.787$ ) and NLR (c-index 0.755, 95\% $\mathrm{CI}=0.701-0.786)$. Of note, the sensitivity analysis using, SII, PLR, and NLR as continuous rather than categorical 


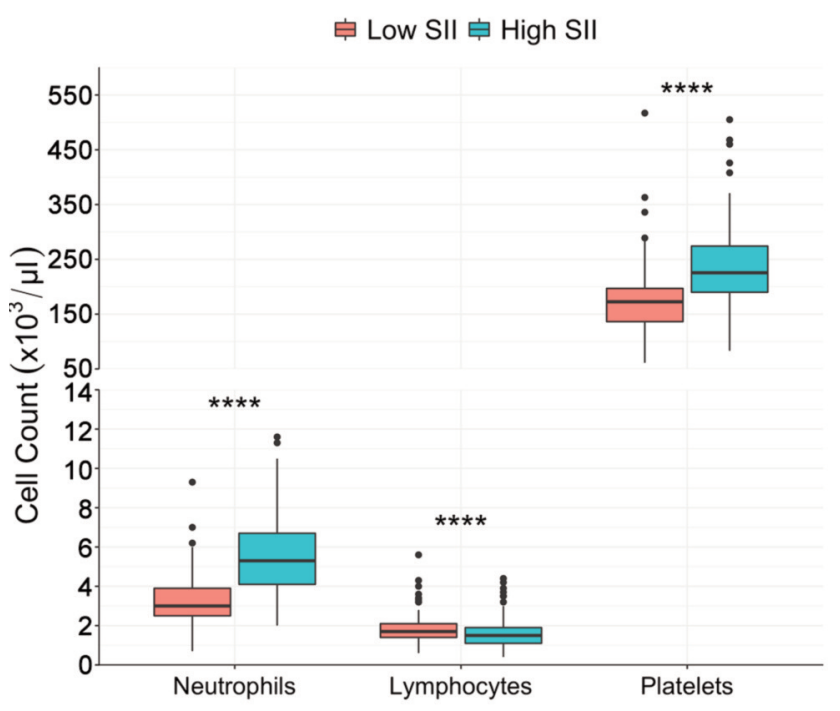

Figure 1. Circulating levels of neutrophils, lymphocytes, and platelets in peripheral blood samples of patients with low versus high systemic immune-inflammation index (SII). High SII was defined as $>475.5$. $* * * *$ Significantly different at $p<0.01$.

variables revealed superior performance of the SII model compared to those incorporating PLR or NLR (Table III).

Among DDKT recipients, only high NLR (>1.6) was associated with DGF, conferring $49 \%$ reduced odds of DGF on unadjusted analysis $(\mathrm{OR}=0.51,95 \% \mathrm{CI}=0.28-0.93$, $p=0.03$ ). When adjusted for age, cold ischemia time, donor type (donor brain death $v s$. donor circulatory death), US Public Health Service increased risk status, and KDPI, high NLR remained associated with a 53\% decrease in the odds of DGF (OR=0.47, 95\% CI=0.25-0.88, $p=0.02)$. Neither high SII (>492.9) nor high PLR (>104.4) were independent predictors of DGF among DDKT recipients (SII: OR=0.63, 95\% CI=0.39-1.03, $p=0.06$; PLR: $\mathrm{OR}=1.68,95 \% \mathrm{CI}=0.99$ $2.89, p=0.057)$. After the addition of each inflammatory index to the model, the model including SII was associated with a c-index of $0.697 \quad(95 \% \quad \mathrm{CI}=0.629-0.738)$, outperforming models including PLR (c-index 0.695, 95\% $\mathrm{CI}=0.612-0.730)$ and NLR (c-index 0.696, 95\% CI=0.6250.737 ). A sensitivity analysis using all inflammatory indices as continuous variables revealed superior performance of the SII model compared to those incorporating PLR or NLR.

Association between inflammatory indices and patient, rejection-free, and graft survival. Patient survival was significantly increased among patients with high SII, high PLR, and high NLR (SII: log-rank $p<0.01$; PLR: log-rank $p<0.01$; NLR: log-rank $p=0.02$ ) (Figures 2-4, part A). Only high SII was associated with improved rejection-free survival (log-rank $p=0.045$ ) (Figure 2B). There was no difference in graft survival between SII, PLR, and NLR groups (SII: $\log$-rank $p=0.2$; PLR: log-rank $p=0.5$; NLR: log-rank $p=0.5$ ) (Figures 2-4, part C). Among DDKT recipients, patient survival was increased among those with high SII and high PLR (SII: log-rank $p=0.02$; PLR: $\log$-rank $p=0.02$ ); there was no difference in patient survival between DDKT recipients with high versus low NLR (log-rank $p=0.5$ ). Rejection-free survival and graft survival were similar among DDKT recipients with high versus low SII, PLR, and NLR (all log-rank $p>0.05$ ).

On multivariable analysis, high SII, high PLR, and high NLR were all independently associated with significantly increased patient survival [SII: hazard ratio $(\mathrm{HR})=0.24,95 \%$ $\mathrm{CI}=0.07-0.76, p=0.02$; PLR: $\mathrm{HR}=0.27,95 \% \mathrm{CI}=0.09-0.75$, $p=0.01$; NLR: $\mathrm{HR}=0.13,95 \% \mathrm{CI}=0.02-0.98, p=0.047]$. After the addition of each inflammatory index, the model including NLR was associated with a c-index of 0.788 (95\% CI=0.6900.832), outperforming models including SII (c-index 0.786, 95\% $\mathrm{CI}=0.648-0.846)$ and PLR (c-index 0.774, 95\% $\mathrm{CI}=0.655-0.834) \quad$ (Table IV). On sensitivity analysis including each inflammatory index as a continuous variable, the SII model demonstrated superior performance compared to the PLR and NLR models. For the entire patient cohort, none of the inflammatory indices were independently associated with rejection-free or graft survival (Table V).

Among DDKT recipients, only high PLR (>104.4) was independently associated with increased patient survival ( $\mathrm{HR}=0.30,95 \% \mathrm{CI}=0.10-0.91, p=0.03)$. The models including SII and PLR demonstrated comparable performance (SII: cindex 0.776 , 95\% CI=0.552-0.842; PLR: 0.776 , 95\% $\mathrm{CI}=0.644-0.840$ ), both outperforming the NLR model (cindex $0.722,95 \% \mathrm{CI}=0.577-0.773$ ). In the sensitivity analysis including each inflammatory index as a continuous variable, the PLR model was superior, demonstrating marginally better performance compared to the SII model. Among DDKT recipients, neither SII nor PLR or NLR were independently associated with rejection-free survival or graft survival.

\section{Discussion}

In this single-institution analysis of patients undergoing primary kidney transplantation, we evaluated the ability of SII, a novel inflammatory biomarker, to predict posttransplant prognosis and compared its prognostic capabilities to those of PLR and NLR. On unadjusted analysis, high SII was associated with reduced odds of DGF and improved patient and rejection-free survival; however, after adjustment high SII was independently associated with improved patient survival alone. Models for the prediction of DGF, patient survival, and rejection-free survival, that included SII as a dichotomous or continuous variable performed well compared to those including only PLR or NLR. Although it remains difficult to identify patients at risk of poor posttransplant prognosis based on SII alone, our study suggests that when considered in combination with other relevant 
in vivo $34: 3349-3360(2020)$

Table III. Bivariable and multivariable analysis of delayed graft function in the whole patient cohort.

\begin{tabular}{|c|c|c|c|c|c|c|c|c|}
\hline \multirow[b]{2}{*}{ Variable } & \multicolumn{2}{|l|}{ Bivariate } & \multicolumn{2}{|c|}{ Multivariable (+SII) } & \multicolumn{2}{|c|}{ Multivariable (+PLR) } & \multicolumn{2}{|c|}{ Multivariable (+NLR) } \\
\hline & OR $(95 \% \mathrm{CI})$ & $p$-Value & OR $(95 \% \mathrm{CI})$ & $p$-Value & OR $(95 \% \mathrm{CI})$ & $p$-Value & OR $(95 \% \mathrm{CI})$ & $p$-Value \\
\hline Age & $1.00(0.98-1.02)$ & 0.84 & & & & & & \\
\hline Gender (female) & $0.45(0.29-0.71)$ & $<0.01$ & $0.47(0.28-0.78)$ & $<0.01$ & $0.46(0.27-0.76)$ & $<0.01$ & $0.44(0.26-0.73)$ & $<0.01$ \\
\hline Race (White) & $0.50(0.31-0.79)$ & $<0.01$ & & & & & & \\
\hline Ethnicity (Hispanic) & $1.74(0.49-5.88)$ & 0.37 & & & & & & \\
\hline PRA class I at transplant & $0.99(0.98-1.00)$ & 0.013 & & & & & & \\
\hline PRA class II at transplant & $0.98(0.97-0.99)$ & 0.011 & $0.99(0.97-1.00)$ & 0.072 & $0.99(0.97-1.00)$ & 0.064 & $0.99(0.97-1.00)$ & 0.080 \\
\hline \multicolumn{9}{|l|}{ Etiology of kidney disease } \\
\hline Other & Ref & & Ref & & Ref & & Ref & \\
\hline Type 2 diabetes mellitus & $2.29(1.31-4.04)$ & $<0.01$ & $2.29(1.24-4.28)$ & $<0.01$ & $2.67(1.44-5.04)$ & $<0.01$ & $2.22(1.19-4.16)$ & 0.012 \\
\hline $\begin{array}{l}\text { Hypertensive } \\
\text { nephrosclerosis }\end{array}$ & $1.71(0.93-3.15)$ & 0.087 & $1.39(0.71-2.73)$ & 0.34 & $1.68(0.86-3.31)$ & 0.13 & $1.37(0.69-2.71)$ & 0.36 \\
\hline Focal glomerular sclerosis & $0.92(0.36-2.18)$ & 0.86 & $0.80(0.29-2.03)$ & 0.65 & $1.00(0.36-2.57)$ & 0.99 & $0.81(0.30-2.06)$ & 0.67 \\
\hline Polycystic kidney disease & $1.32(0.57-2.94)$ & 0.51 & $1.21(0.48-2.94)$ & 0.68 & $1.36(0.54-3.30)$ & 0.51 & $1.30(0.52-3.16)$ & 0.56 \\
\hline Pre-transplant malignancy & $1.07(0.51-2.14)$ & 0.85 & & & & & & \\
\hline Cold ischemic time & $1.001(1.000-1.001)$ & $<0.01$ & $1.000(1.000-1.001)$ & 0.29 & $1.000(1.000-1.001)$ & 0.20 & $1.000(1.000-1.001)$ & 0.27 \\
\hline Warm ischemic time & $1.05(1.02-1.08)$ & $<0.01$ & & & & & & \\
\hline Machine perfusion used & $2.83(1.66-5.04)$ & $<0.01$ & & & & & & \\
\hline \multicolumn{9}{|l|}{ Donor type } \\
\hline $\mathrm{DBD}$ & Ref & & Ref & & Ref & & Ref & \\
\hline DCD & $2.36(1.44-3.89)$ & $<0.01$ & $2.37(1.39-4.08)$ & $<0.01$ & $2.41(1.41-4.16)$ & $<0.01$ & $2.33(1.37-3.99)$ & $<0.01$ \\
\hline Living & $0.088(0.014-0.29)$ & $<0.01$ & $0.10(0.015-0.43)$ & $<0.01$ & $0.10(0.015-0.43)$ & $<0.01$ & $0.11(0.015-0.44)$ & $<0.01$ \\
\hline PHS increased risk & $0.55(0.33-0.90)$ & 0.020 & & & & & & \\
\hline Extended criteria donor & $1.61(0.88-2.95)$ & 0.12 & & & & & & \\
\hline KDPI & $1.01(1.00-1.02)$ & 0.088 & & & & & & \\
\hline HLA mismatch & $1.24(1.06-1.47)$ & $<0.01$ & & & & & & \\
\hline \multicolumn{9}{|l|}{ Graft laterality } \\
\hline Left & Ref & & & & & & & \\
\hline Right & $1.68(1.08-2.61)$ & 0.021 & & & & & & \\
\hline Dual & $3.63(0.78-18.98)$ & 0.099 & & & & & & \\
\hline SII $>475.5$ & $0.60(0.39-0.92)$ & 0.020 & $0.64(0.39-1.04)$ & 0.074 & & & & \\
\hline PLR $>103.4$ & $1.49(0.92-2.46)$ & 0.11 & & & $1.92(1.12-3.33)$ & 0.019 & & \\
\hline $\mathrm{NLR}>3.3$ & $0.60(0.37-0.94)$ & 0.029 & & & & & $0.65(0.38-1.09)$ & 0.10 \\
\hline C-Index ${ }^{a}$ & & & $0.755(0.704-0.787)$ & & $0.761(0.693-0.792)$ & & $0.755(0.701-0.786)$ & \\
\hline C-Index a,b & & & $0.751(0.688-0.783)$ & & $0.749(0.677-0.780)$ & & $0.749(0.679-0.780)$ & \\
\hline
\end{tabular}

IQR: Interquartile range; NLR: neutrophil to lymphocyte ratio; PLR: platelet to lymphocyte ratio; DBD: donors from brain death; DCD: donor from circulatory death; PHS: public health service; HLA: human leukocyte antigen; KDPI; kidney donor profile index; OR: odds ratio; SII: systemic immune-inflammation index; PRA: pre-transplant panel reactive antibody Statistically significant $p$-values are shown in bold. ${ }^{\mathrm{a} C}$-Index $(95 \%$ confidence interval) determined based on 1,000 bootstrapped resamples. bSII, PLR, and NLR used as continuous rather than categorical variables. Statistically significant $p$-values are shown in bold.

clinical parameters, SII may represent a useful non-invasive prognostic biomarker for use in kidney transplantation.

The association between inflammation and suboptimal outcomes after kidney transplantation is well-documented. In particular, the downstream activation and differentiation of recipient lymphocytes during ischemia-reperfusion injury contributes to immediate allograft injury underlying DGF and stimulates development of a lymphocyte-mediated response to foreign allograft tissue that later manifests as $\operatorname{AR}(3,25)$. Several recent studies examining the impact of induction immunosuppression on post-transplant prognosis found reduced rates of DGF and AR as well as improved long-term patient and graft survival among kidney transplant recipients receiving lymphocyte-depleting agents, further highlighting the role of circulating lymphocytes in determining both early and late post-transplant outcomes $(26,27)$. In the present study, low SII was driven in part by significantly increased levels of circulating lymphocytes in peripheral blood samples, suggesting that relative elevation of lymphocytes may underlie the association between low SII and development of DGF and reduced patient and rejection-free survival. Although our findings stand in contrast to those employing SII for prognostication in cancer (19-22), the inflammatory response to cancer entails an increase in neutrophils and 
A

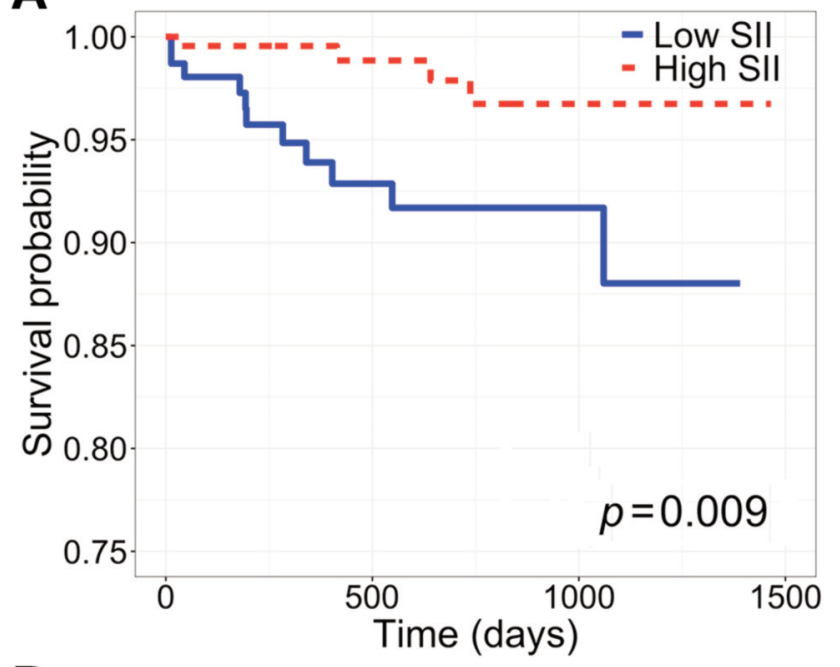

B

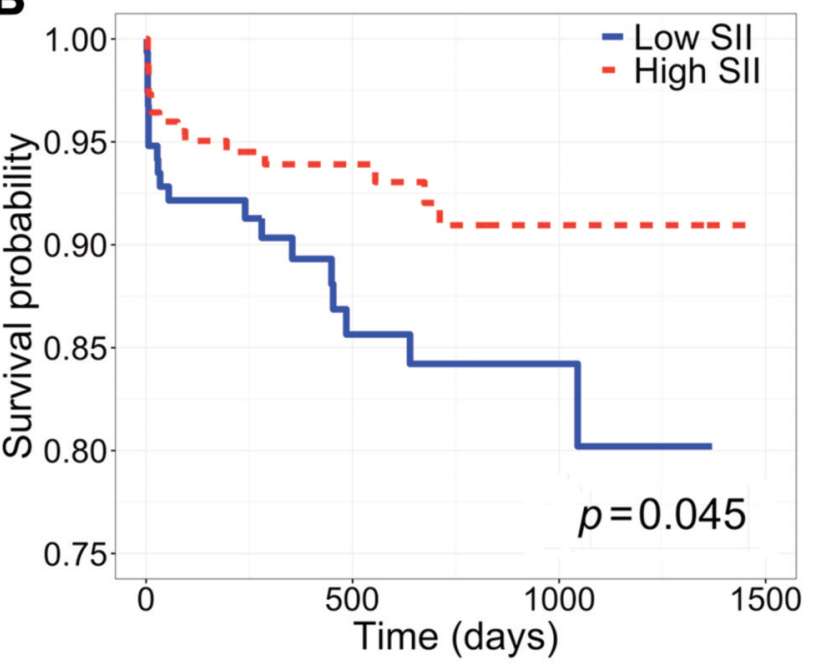

C

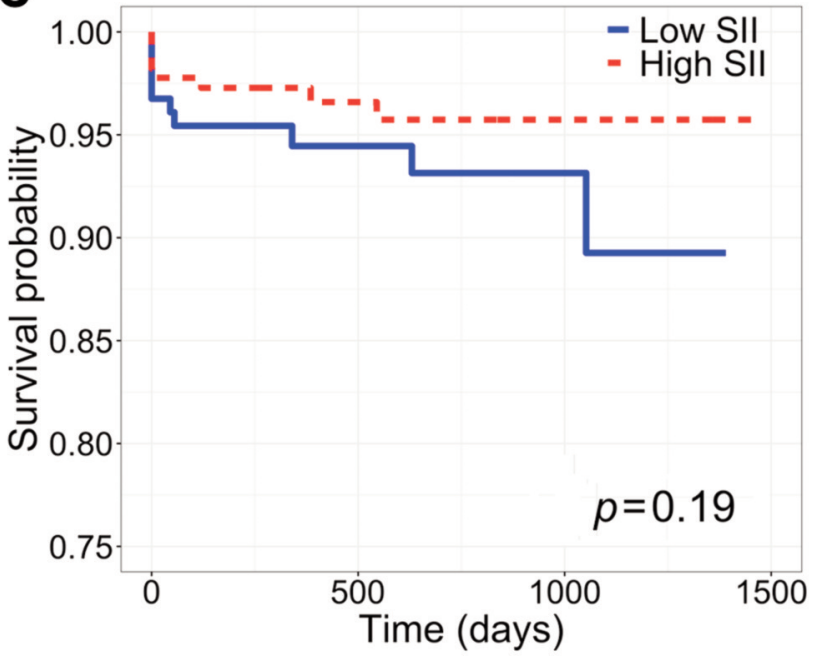

Figure 2. Kaplan-Meier survival analysis of the whole cohort stratified by high versus low systemic immune-inflammation index (SII). A: Patient survival. B: Rejection-free survival. C: Graft survival.

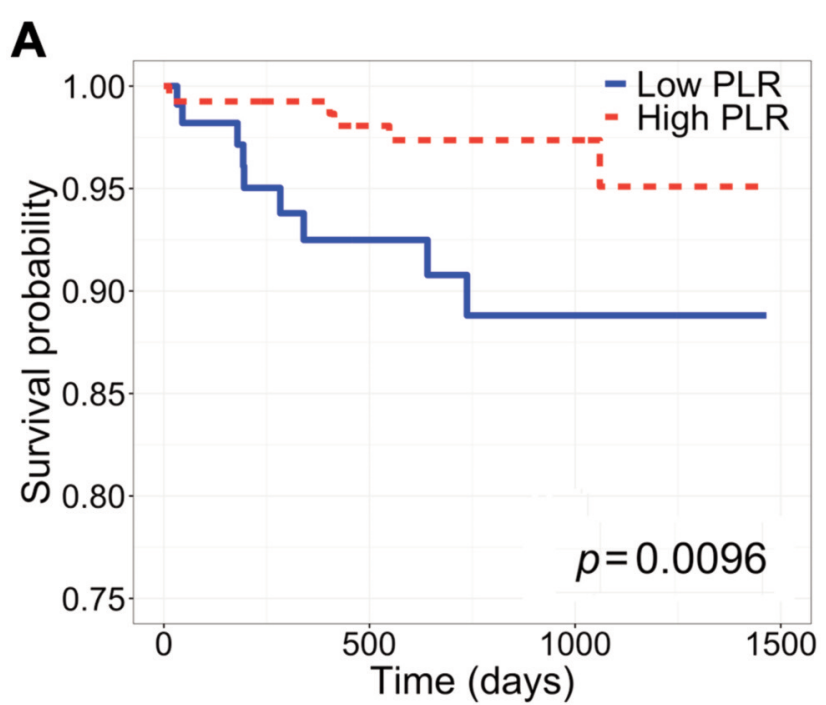

B

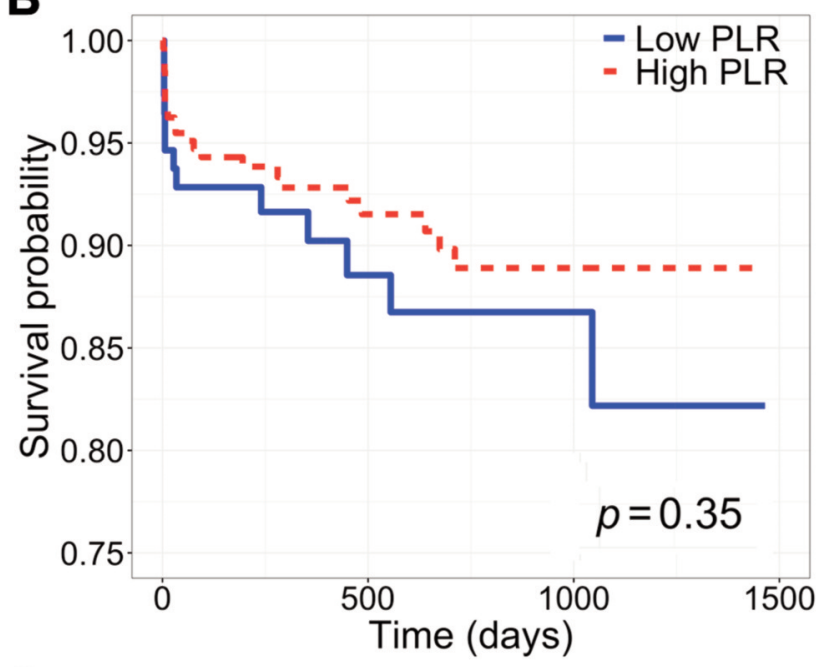

C

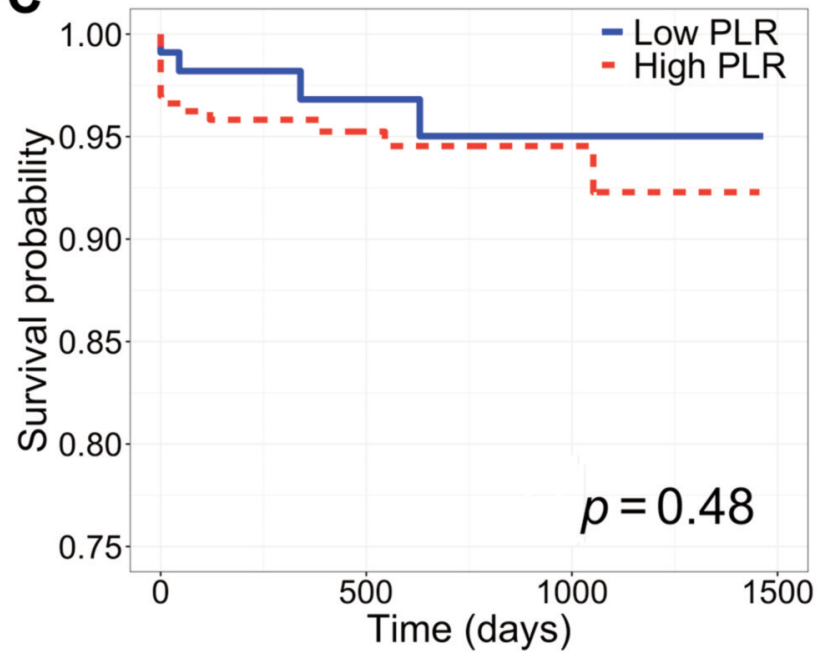

Figure 3. Kaplan-Meier survival analysis of the whole cohort stratified by high versus low platelet-to-lymphocyte ratio (PLR). A: Patient survival. B: Rejection-free survival. C: Graft survival. 

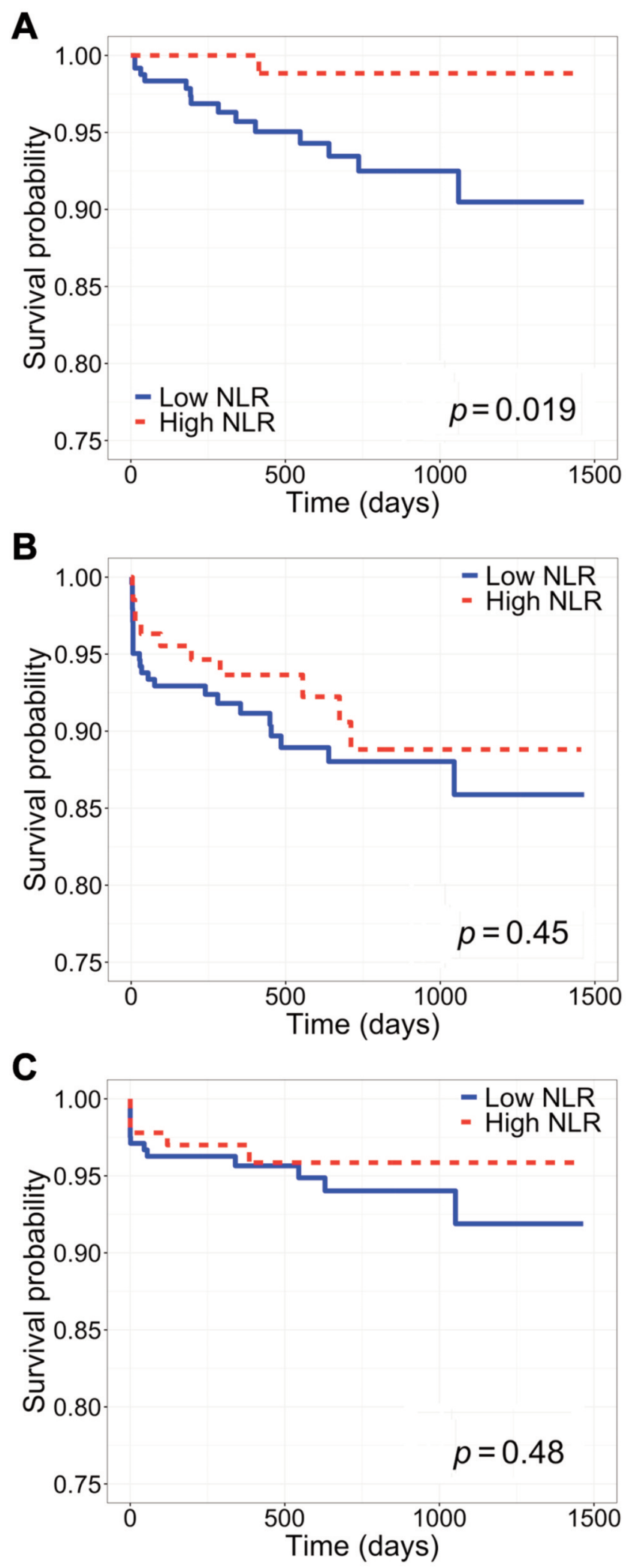

Figure 4. Kaplan-Meier survival analysis of the whole cohort stratified by high versus low neutrophil-to-lymphocyte ratio (NLR). A: Patient survival. B: Rejection-free survival. C: Graft survival. platelets with a concurrent reduction in lymphocytes (9), suggesting that the underlying pathophysiology of cancer versus organ rejection may explain the contrast in these findings, and may also dictate the expected associations between inflammatory biomarkers and outcomes among diverse conditions.

To our knowledge, this is the first study to apply SII to prognostication in kidney transplantation, however, inflammatory biomarkers including NLR and PLR have previously been used in this setting. Interestingly, several institutional cohort studies examining the associations between NLR and PLR and DGF and AR remain divided in their findings (14-18). Among those studies reporting an association between high NLR and PLR and the development of immunological injury, the primary cited mechanism involves neutrophilia and thrombocytosis occurring during ischemia-reperfusion injury, both of which contribute to the formation of immunogenic microthrombi within the renal vasculature $(3,14,15,25)$. Conversely, those studies reporting increased incidence of DGF and AR among patients with low NLR and PLR attributed their findings to a relative increase in circulating lymphocytes $(16,17)$. Our findings contribute to both sides of this debate, demonstrating independent associations between high PLR and DGF and between high NLR and improved patient survival. In the context of the preceding studies and the known pathophysiology of ischemia-reperfusion injury and allograft immunogenicity, the association between high PLR, increased circulating platelets, and DGF, suggests that the formation of platelet microthrombi may be a more significant contributor to early allograft injury, whereas low NLR, a relatively elevated level of lymphocytes, and poor long-term prognosis further emphasize the central role of a lymphocyte-mediated response in ongoing allograft injury over time.

The pre-transplant inflammatory profile of kidney transplant recipients may be attributed in part to chronic inflammation resulting from prolonged dialysis dependence (28). In a cohort study of patients with ESRD on hemodialysis, Yaprak and colleagues found that high NLR and PLR were associated with increased all-cause mortality, supporting the association between systemic inflammation and poor prognosis in this group (12). Prolonged pretransplant dialysis is a known risk factor for reduced patient and graft survival $(29,30)$, partially explaining the transplant community's preference for LDKT, which, when available, provides timelier access to transplantation and reduced time on dialysis $(2,30)$. However, in our study, both NLR and PLR were significantly higher among LDKT recipients compared to DDKT recipients, which, in the context of the study by Yaprak et al., suggests that we might expect worse prognosis in this patient subgroup. However, in LDKT recipients specifically, pre-transplant alloreactivity - antigen recognition by recipient lymphocytes - and lymphocyte 
Table IV. Bivariable and multivariable analysis of patient survival in the whole patient cohort.

\begin{tabular}{|c|c|c|c|c|c|c|c|c|}
\hline \multirow[b]{2}{*}{ Variable } & \multicolumn{2}{|l|}{ Bivariate } & \multicolumn{2}{|c|}{ Multivariable (+SII) } & \multicolumn{2}{|c|}{ Multivariable (+PLR) } & \multicolumn{2}{|c|}{ Multivariable (+NLR) } \\
\hline & HR $(95 \%$ CI $)$ & $p$-Value & HR $(95 \%$ CI $)$ & $p$-Value & HR $(95 \%$ CI $)$ & $p$-Value & $\mathrm{HR}(95 \% \mathrm{CI})$ & $p$-Value \\
\hline Age & $1.06(1.00-1.11)$ & 0.047 & $1.06(1.01-1.12)$ & 0.031 & $1.06(1.00-1.12)$ & 0.039 & $1.06(1.00-1.12)$ & 0.033 \\
\hline Gender (female) & $0.61(0.21-1.77)$ & 0.36 & & & & & & \\
\hline Race (White) & $0.40(0.11-1.41)$ & 0.15 & & & & & & \\
\hline Ethnicity (Hispanic) & $2.92(0.38-22.3)$ & 0.30 & & & & & & \\
\hline PRA Class I at transplant & $1.00(0.98-1.02)$ & 0.96 & & & & & & \\
\hline PRA Class II at transplant & $1.00(0.97-1.02)$ & 0.77 & $1.00(0.97-1.03)$ & 0.86 & $0.99(0.97-1.02)$ & 0.63 & $1.00(0.97-1.02)$ & 0.74 \\
\hline \multicolumn{9}{|l|}{ Etiology of kidney disease } \\
\hline Other & Ref & & & & & & & \\
\hline Type 2 diabetes mellitus & $1.19(0.34-4.12)$ & 0.79 & & & & & & \\
\hline $\begin{array}{l}\text { Hypertensive } \\
\text { nephrosclerosis }\end{array}$ & $1.07(0.25-4.46)$ & 0.93 & & & & & & \\
\hline Focal glomerular sclerosis & $1.71(0.33-8.83)$ & 0.52 & & & & & & \\
\hline Polycystic kidney disease & - & - & & & & & & \\
\hline Pre-transplant malignancy & $2.70(0.76-9.58)$ & 0.13 & & & & & & \\
\hline Cold ischemic time & $1.000(1.000-1.001)$ & 0.99 & & & & & & \\
\hline Warm ischemic time & $1.03(0.96-1.10)$ & 0.44 & & & & & & \\
\hline Machine perfusion used & $0.85(0.29-2.50)$ & 0.78 & & & & & & \\
\hline \multicolumn{9}{|l|}{ Donor type } \\
\hline DBD & Ref & & Ref & & Ref & & Ref & \\
\hline DCD & $0.20(0.026-1.54)$ & 0.12 & $0.18(0.024-1.42)$ & 0.10 & $0.15(0.020-1.20)$ & 0.075 & $0.17(0.022-1.33)$ & 0.091 \\
\hline Living & $0.68(0.15-3.05)$ & 0.62 & $0.81(0.18-3.64)$ & 0.78 & $0.77(0.17-3.49)$ & 0.74 & $0.80(0.18-3.63)$ & 0.78 \\
\hline PHS increased risk & $1.06(0.33-3.45)$ & 0.92 & & & & & & \\
\hline Extended criteria donor & $2.71(0.83-8.80)$ & 0.098 & & & & & & \\
\hline KDPI & $1.01(0.99-1.03)$ & 0.41 & & & & & & \\
\hline HLA mismatch & $1.17(0.80-1.73)$ & 0.42 & & & & & & \\
\hline \multicolumn{9}{|l|}{ Graft laterality } \\
\hline Left & Ref & & & & & & & \\
\hline Right & $0.57(0.19-1.71)$ & 0.32 & & & & & & \\
\hline Dual & $3.44(0.43-27.29)$ & 0.24 & & & & & & \\
\hline $\mathrm{SII}>475.5$ & $0.24(0.078-0.77)$ & 0.016 & $0.24(0.074-0.76)$ & 0.016 & & & & \\
\hline PLR $>103.4$ & $0.28(0.099-0.78)$ & 0.015 & & & $0.27(0.094-0.75)$ & 0.012 & & \\
\hline NLR $>3.3$ & $0.13(0.017-0.98)$ & 0.047 & & & & & $0.13(0.017-0.98)$ & 0.047 \\
\hline C-Index ${ }^{a}$ & & & $0.786(0.648-0.846)$ & & $0.774(0.655-0.834)$ & & $0.788(0.690-0.832)$ & \\
\hline C-Index $a, b$ & & & $0.817(0.654-0.873)$ & & $0.792(0.693-0.833)$ & & $0.771(0.649-0.826)$ & \\
\hline
\end{tabular}

IQR: Interquartile range; NLR: neutrophil to lymphocyte ratio; PLR: platelet to lymphocyte ratio; DBD: donors from brain death; DCD: donor from circulatory death; PHS: public health service; HLA: human leukocyte antigen; KDPI; kidney donor profile index; OR: odds ratio; SII: systemic immune-inflammation index; PRA: pre-transplant panel reactive antibody. ${ }^{\mathrm{a} C} \mathrm{C}-\mathrm{Index}$ (95\% confidence interval) determined based on 1,000 bootstrapped resamples. bSII, PLR, and NLR used as continuous rather than categorical variables. Statistically significant $p$-values are shown in bold.

activation have been found to contribute to DGF and the occurrence of AR during the first year after transplantation $(31,32)$, suggesting that recipient lymphocytes are of particular importance in this population where the degree of ischemia-reperfusion injury is lessened by reduced cold ischemia times and often improved allograft quality compared to DDKT. Although our ability to evaluate the association between inflammatory biomarkers and post-transplant prognosis among LDKT recipients was limited by the small sample size, in the context of our study as a whole, we can infer that higher NLR and PLR among LDKT recipients may reflect a reduction in circulating lymphocytes, aligning with a reduced incidence of DGF in this subgroup and possibly portending improved long-term post-transplant prognosis. Additionally, in a recent study, Azab et al. found that on average, NLR was significantly higher among non-Hispanic white patients compared to all other racial groups (33), suggesting that our findings may be attributed in part to the predominantly white racial composition of the LDKT group.

In light of the complex pathophysiology of immediate and delayed immunologic injury post-kidney transplantation, the use of SII as a holistic marker of inflammation and the relative contributions of different immune pathways may be a valuable addition to the field of transplantation. Overcoming the inconsistencies in NLR and PLR highlighted by the conflicting associations in our study and others by 
in vivo $34: 3349-3360(2020)$

Table V. Bivariable and multivariable analysis of rejection-free survival in the whole patient cohort.

\begin{tabular}{|c|c|c|c|c|c|c|c|c|}
\hline \multirow[b]{2}{*}{ Variable } & \multicolumn{2}{|l|}{ Bivariate } & \multicolumn{2}{|c|}{ Multivariable (+SII) } & \multicolumn{2}{|c|}{ Multivariable (+PLR) } & \multicolumn{2}{|c|}{ Multivariable (+NLR) } \\
\hline & HR $(95 \% \mathrm{CI})$ & $p$-Value & $\operatorname{HR}(95 \% \mathrm{CI})$ & $p$-Value & HR $(95 \% \mathrm{CI})$ & $p$-Value & $\operatorname{HR}(95 \% \mathrm{CI})$ & $p$-Value \\
\hline Age & $0.97(0.95-0.99)$ & 0.017 & $0.97(0.95-1.00)$ & 0.022 & $0.97(0.95-0.99)$ & 0.014 & $0.97(0.95-0.99)$ & 0.017 \\
\hline Gender (female) & $0.88(0.46-1.72)$ & 0.72 & & & & & & \\
\hline Race (White) & $0.79(0.40-1.59)$ & 0.51 & & & & & & \\
\hline Ethnicity (Hispanic) & - & - & & & & & & \\
\hline PRA Class I at transplant & $0.99(0.98-1.01)$ & 0.47 & & & & & & \\
\hline PRA Class II at transplant & $0.98(0.96-1.01)$ & 0.20 & & & & & & \\
\hline \multicolumn{9}{|l|}{ Etiology of kidney disease } \\
\hline Other & Ref & & & & & & & \\
\hline Type 2 diabetes mellitus & $0.73(0.30-1.76)$ & 0.48 & & & & & & \\
\hline $\begin{array}{l}\text { Hypertensive } \\
\text { nephrosclerosis }\end{array}$ & $1.36(0.59-3.10)$ & 0.47 & & & & & & \\
\hline Focal glomerular sclerosis & $0.58(0.13-2.56)$ & 0.47 & & & & & & \\
\hline Polycystic kidney disease & $0.80-0.23-2.79)$ & 0.72 & & & & & & \\
\hline Pre-transplant malignancy & $0.58(0.14-2.40)$ & 0.45 & & & & & & \\
\hline Cold ischemic time & $1.000(1.000-1.001)$ & 0.18 & & & & & & \\
\hline Warm ischemic time & $1.00(0.96-1.05)$ & 0.96 & & & & & & \\
\hline Machine perfusion used & $1.76(0.77-4.02)$ & 0.18 & & & & & & \\
\hline \multicolumn{9}{|l|}{ Donor type } \\
\hline $\mathrm{DBD}$ & Ref & & & & & & & \\
\hline DCD & $1.76(0.87-3.53)$ & 0.11 & & & & & & \\
\hline Living & $0.63(0.19-2.14)$ & 0.46 & & & & & & \\
\hline PHS increased risk & $1.10(0.53-2.27)$ & 0.80 & & & & & & \\
\hline Extended criteria donor & $1.01(0.39-2.62)$ & 0.98 & & & & & & \\
\hline KDPI & $1.00(0.99-1.02)$ & 0.85 & & & & & & \\
\hline HLA mismatch & $1.32(1.02-1.73)$ & 0.039 & & & & & & \\
\hline \multicolumn{9}{|l|}{ Graft laterality } \\
\hline Left & Ref & & & & & & & \\
\hline Right & $1.17(0.61-2.25)$ & 0.64 & & & & & & \\
\hline Dual & - & - & & & & & & \\
\hline SII $>475.5$ & $0.52(0.27-1.00)$ & 0.049 & $0.53(0.28-1.03)$ & 0.061 & & & & \\
\hline PLR $>103.4$ & $0.72(0.37-1.43)$ & 0.35 & & & $0.68(0.34-1.34)$ & 0.26 & & \\
\hline NLR $>3.3$ & $0.76(0.37-1.54)$ & 0.45 & & & & & $0.75(0.37-1.52)$ & 0.42 \\
\hline C-Index ${ }^{a}$ & & & $0.635(0.530-0.707)$ & & $0.611(0.518-0.685)$ & & $0.607(0.514-0.680)$ & \\
\hline C-Index ${ }^{a, b}$ & & & $0.624(0.503-0.703)$ & & $0.612(0.515-0.692)$ & & $0.592(0.488-0.663)$ & \\
\hline
\end{tabular}

IQR: Interquartile range; NLR: neutrophil to lymphocyte ratio; PLR: platelet to lymphocyte ratio; DBD: donors from brain death; DCD: donor from circulatory death; PHS: public health service; HLA: human leukocyte antigen; KDPI; kidney donor profile index; OR: odds ratio; SII: systemic immune-inflammation index; PRA: pre-transplant panel reactive antibody. ${ }^{\mathrm{a} C} \mathrm{C}-\mathrm{Index}$ ( $95 \%$ confidence interval) determined based on 1,000 bootstrapped resamples. bSII, PLR, and NLR used as continuous rather than categorical variables. Statistically significant $p$-values are shown in bold.

incorporating neutrophil, lymphocyte, and platelet counts in a single metric may offer a more robust indication of the state of the recipient immune system at the time of transplantation. Likewise, predictive models incorporating SII demonstrated robust prognostic capabilities, particularly with regard to DGF and patient survival in which model c-statistics exceeded 0.750 . However, given that our models still performed well when SII was included as a continuous covariate, the true utility of SII in kidney transplantation may be in placing patients along a continuum of relative immune contributions rather than into high or low strata. Indeed, classifying patients into high and low groups based on a single biomarker remains difficult, particularly when considering diverse patient groups across institutions and throughout time (34). Furthermore, the concept of 'normal' SII is undefined and the interest in this setting rests less with whether patients have objectively higher or lower SII compared to normal, but with how particular levels of inflammation and immune activation relate to the expected post-transplant course.

There are several limitations to our study that warrant discussion. Firstly, this study examined a cohort of kidney transplant recipients at a single academic institution, which may limit its generalizability. However, among studies exploring the use of inflammatory biomarkers in kidney transplantation, ours is the largest to date, providing considerable new data to this area of investigation. Additionally, patients undergoing repeat 
kidney transplantation were excluded from our study due to concerns regarding confounding of inflammatory indices by pre-transplant immunosuppression; we were therefore unable to ascertain the prognostic utility of SII among the broader population of kidney transplant recipients including those who have undergone prior transplantation. Finally, the association between inflammatory indices and post-transplant outcomes was assessed using pre-transplant data from a single timepoint. Among patients in our study, the particular time of blood sample collection varied as LDKT recipients may have had pretransplant blood drawn at a pre-operative evaluation visit prior to the transplant hospital admission, which may have been weeks up to 6 months prior to transplantation, versus DDKT recipients who routinely have blood drawn at the time of admission, typically within 24 hours pre-transplant. Additionally, given the dynamic nature of immune activation, data taken from multiple pre-transplant timepoints may provide a better understanding of patients' immune systems and how they may respond to transplantation. However, with the goal of applying inflammatory indices such as SII to clinical practice, collation of data across multiple pre-transplant time points may not be feasible at the institutional or national level that will be needed to validate this metric in a large multi-institutional study.

\section{Conclusion}

In this single-institution analysis of primary kidney transplant recipients, we found that high SII was associated with improved post-transplant prognosis, including reduced odds of DGF and improved patient and rejection-free survival. While SII was not an independent predictor of immunologic injury, when used in combination with other known risk factors for poor posttransplant prognosis, SII was a powerful predictor of DGF and patient survival following kidney transplant. Given the widespread availability of this safe and non-invasive inflammatory biomarker, determination of SII in the pretransplant setting may improve prognostication to tailor pretransplant decision-making for kidney transplantation recipients in order to optimize both early and late post-transplant outcomes.

\section{Conflicts of Interest}

The Authors report no conflicts of interest. SEH is supported by a Pfizer Foundation grant and the Duke Clinical Translational Science Institute. The content is solely the responsibility of the Authors and does not necessarily represent the official views of the Pfizer Foundation or Duke Clinical Translational Science Institute.

\section{Authors' Contributions}

Samantha E. Halpern: Conception and study design, data collection, analysis and interpretation of data, drafting and critically revising the article, final approval of the version to be published. Dimitrios Moris: Conception and study design, data collection, analysis and interpretation of data, drafting and critically revising the article, final approval of the version to be published. Brian I. Shaw: Conception and study design, analysis and interpretation of data, critically revising the article, final approval of the version to be published. Madison K. Krischak: Data collection, critically revising the article, final approval of the version to be published. Danae G. Olaso: Data collection, critically revising the article, final approval of the version to be published. Samuel J. Kesseli: Conception and study design, critically revising the article, final approval of the version to be published. Kadiyala Ravindra: Conception and study design, critically revising the article, final approval of the version to be published. Lisa M. McElroy: Critically revising the article, final approval of the version to be published. Andrew S. Barbas: Conception and study design, analysis and interpretation of data, critically revising the article, final approval of the version to be published.

\section{References}

1 Tonelli M, Wiebe N, Knoll G, Bello A, Browne S, Jadhav D, Klarenbach $\mathrm{S}$ and Gill J: Systematic review: kidney transplantation compared with dialysis in clinically relevant outcomes. Am J Transplant 11: 2093-2109, 2011. PMID: 21883901. DOI: 10.1111/j.1600-6143.2011.03686.x

2 Hart A, Smith JM, Skeans MA, Gustafson SK, Wilk AR, Castro S, Foutz J, Wainright JL, Snyder JJ, Kasiske BL and Israni AK: OPTN/SRTR 2018 Annual Data Report: kidney. Am J Transplant 20: 20-130, 2020. PMID: 31898417. DOI: 10.1111/ajt.15672

3 Perico N, Cattaneo D, Sayegh MH and Remuzzi G: Delayed graft function in kidney transplantation. Lancet 364: 1814-1827, 2004. PMID: 15541456. DOI: 10.1016/S0140-6736(04)17406-0

4 Singh N, Samant H, Hawxby A and Samaniego MD: Biomarkers of rejection in kidney transplantation. Curr Opin Organ Transplant 24: 103-110, 2019. PMID: 30540576. DOI: 10.1097/ MOT.0000000000000606

5 Menon MC, Murphy B and Heeger PS: Moving biomarkers toward clinical implementation in kidney transplantation. J Am Soc Nephrol 28: 735-747, 2017. PMID: 28062570. DOI: 10.1681/ASN.2016080858

6 Van Loon E and Naesens M: Do we need noninvasive biomarkers for delayed graft function after kidney transplantation? Transplantation 103: 870-872, 2019. PMID: 30299375. DOI: 10.1097/TP.0000000000002473

7 Naesens $M$ and Anglicheau D: Precision transplant medicine: Biomarkers to the rescue. J Am Soc Nephrol 29: 24-34, 2018. PMID: 28993504. DOI: 10.1681/ASN.2017010004

8 Templeton AJ, McNamara MG, Šeruga B, Vera-Badillo FE, Aneja P, Ocaña A, Leibowitz-Amit R, Sonpavde G, Knox JJ, Tran B, Tannock IF and Amir E: Prognostic role of neutrophilto-lymphocyte ratio in solid tumors: A systematic review and meta-analysis. J Natl Cancer Inst 106: 1-11, 2014. PMID: 24875653. DOI: $10.1093 /$ jnci/dju124

9 Dupré A and Malik HZ: Inflammation and cancer: What a surgical oncologist should know. Eur J Surg Oncol 44: 566-570, 2018. PMID: 29530345. DOI: 10.1016/j.ejso.2018.02.209

10 Kurtul A and Ornek E: Platelet to lymphocyte ratio in cardiovascular diseases: A systematic review. Angiology 70: 802-818, 2019. PMID: 31030530. DOI: 10.1177/0003319 719845186

11 Dong CH, Wang ZM and Chen SY: Neutrophil to lymphocyte ratio predict mortality and major adverse cardiac events in acute 
coronary syndrome: A systematic review and meta-analysis. Clin Biochem 52: 131-136, 2018. PMID: 29132766. DOI: 10.1016/ j.clinbiochem.2017.11.008

12 Yaprak M, Turan MN, Dayanan R, Akın S, Değirmen E, Yıldırım $M$ and Turgut F: Platelet-to-lymphocyte ratio predicts mortality better than neutrophil-to-lymphocyte ratio in hemodialysis patients. Int Urol Nephrol 48: 1343-1348, 2016. PMID: 27118565. DOI: 10.1007/s11255-016-1301-4

13 Kwon HM, Moon YJ, Jung KW, Park YS, Jun IG, Kim SO, Song JG and Hwang GS: Neutrophil-to-lymphocyte ratio is a predictor of early graft dysfunction following living donor liver transplantation. Liver Int 39: 1545-1556, 2019. PMID: 30903725. DOI: $10.1111 /$ liv.14103

14 Halazun KJ, Marangoni G, Hakeem A, Fraser SM, Farid SG and Ahmad N: Elevated preoperative recipient neutrophil-lymphocyte ratio is associated with delayed graft function following kidney transplantation. Transplant Proc 45: 3254-3257, 2013. PMID: 24182795. DOI: 10.1016/j.transproceed.2013.07.065

15 Baral D, Yang Y, Katwal G, Li S, Wang S, Fan X, Wang Y and Ye Q: Recipient pre-operative neutrophil lymphocyte ratio better predicts delayed graft function than platelet lymphocyte ratio in donation after brain death kidney transplantation. Med J Pokhara Acad Heal Sci 2: 209-216, 2019. DOI: 10.3126/mjpahs.v2i2.28194

16 Hogendorf P, Suska A, Skulimowski A, Rut J, Grochowska M, Wencel A, Dziwisz F, Nowicki M, Szymański D, Poznańska G, Durczyński A and Strzelczyk J: Neutrophil-lymphocyte ratio and creatinine reduction ratio predict good early graft function among adult cadaveric donor renal transplant recipients. Singleinstitution series. Pol Prz Chir Polish J Surg 90: 28-33, 2018. DOI: $10.5604 / 01.3001 .0011 .7499$

17 Naranjo M, Agrawal A, Goyal A and Rangaswami J: Neutrophilto-lymphocyte ratio and platelet-to-lymphocyte ratio predict acute cellular rejection in the kidney allograft. Ann Transplant 23: 467474, 2018. PMID: 29987271. DOI: 10.12659/AOT.909251

18 Ergin G, Değer SM, Köprü B, Derici Ü and Arinsoy T: High neutrophil-to-lymphocyte ratio predicts acute allograft rejection in kidney transplantation: A retrospective study. Turkish J Med Sci 49: 525-530, 2019. PMID: 30834734. DOI: 10.3906/sag-1811-41

19 Hu B, Yang XR, Xu Y, Sun YF, Sun C, Guo W, Zhang X, Wang WM, Qiu SJ, Zhou J and Fan J: Systemic immune-inflammation index predicts prognosis of patients after curative resection for hepatocellular carcinoma. Clin Cancer Res 20: 6212-6222, 2014. PMID: 25271081. DOI: 10.1158/1078-0432.CCR-14-0442

20 Zhong J-H, Huang D-H and Chen Z-Y: Prognostic role of systemic immune-inflammation index in solid tumors: A systematic review and meta-analysis. Oncotarget 8: 75381-75388, 2017. PMID: 29088873. DOI: 10.18632/oncotarget.18856

21 Tsilimigras DI, Moris D, Mehta R, Paredes AZ, Sahara K, Guglielmi A, Aldrighetti L, Weiss M, Bauer TW, Alexandrescu S, Poultsides GA, Maithel SK, Marques HP, Martel G, Pulitano C, Shen F, Soubrane O, Koerkamp BG, Endo I and Pawlik TM: The systemic immune-inflammation index predicts prognosis in intrahepatic cholangiocarcinoma: An international multi-institutional analysis. Hpb, 2020. PMID: 32265108. DOI: 10.1016/j.hpb.2020.03.011

22 Fu H, Zheng J, Cai J, Zeng K, Yao J, Chen L, Li H, Zhang J, Zhang Y, Zhao $\mathrm{H}$ and Yang Y: Systemic Immune-Inflammation Index (SII) is useful to predict survival outcomes in patients after liver transplantation for hepatocellular carcinoma within Hangzhou criteria. Cell Physiol Biochem 47: 293-301, 2018. PMID: 29768257. DOI: 10.1159/000489807
23 Barrio I, Arostegui I, Rodríguez-Álvarez MX and Quintana JM: A new approach to categorising continuous variables in prediction models: Proposal and validation. Stat Methods Med Res 26: 2586-2602, 2017. PMID: 26384514. DOI: $10.1177 /$ 0962280215601873

24 Barrio I, Rodríguez-Álvarez MX and Arostegui I: Optimal Categorisation of Continuous Variables in Prediction Models. 2017. Available at: https://cran.r-project.org/web/packages/ CatPredi/CatPredi.pdf [Last accessed May 11, 2020]

25 Siedlecki A, Irish W and Brennan DC: Delayed graft function in the kidney transplant. Am J Transplant 11: 2279-2296, 2011. PMID: 21929642. DOI: 10.1111/j.1600-6143.2011.03754.x

26 Ravindra K V., Sanoff S, Vikraman D, Zaaroura A, Nanavati A, Sudan D and Irish W: Lymphocyte depletion and risk of acute rejection in renal transplant recipients at increased risk for delayed graft function. Am J Transplant 19: 781-789, 2019. PMID: 30171800. DOI: 10.1111/ajt.15102

27 Weissenbacher A, Hautz T, Kimelman M, Oberhuber R, Ulmer H, Bösmüller C, Maglione M and Schneeberger S: Lymphocytes as an indicator for initial kidney function: A single center analysis of outcome after alemtuzumab or basiliximab induction. J Immunol Res 2015, 2015. PMID: 26171403. DOI: 10.1155/2015/985460

28 Cobo G, Lindholm B and Stenvinkel P: Chronic inflammation in end-stage renal disease and dialysis. Nephrol Dial Transplant 33: iii35-iii40, 2018. PMID: 30281126. DOI: 10.1093/ndt/gfy 175

29 Meier-Kriesche HU, Port FK, Ojo AO, Rudich SM, Hanson JA, Cibrik DM, Leichtman AB and Kaplan B: Effect of waiting time on renal transplant outcome. Kidney Int 58: 1311-1317, 2000. PMID: 10972695. DOI: 10.1046/j.1523-1755.2000.00287.x

30 Foley DP and Sawinski D: Personalizing donor kidney selection choosing the right donor for the right recipient. Clin J Am Soc Nephrol 15: 418-420, 2020. PMID: 31848155. DOI: 10.2215/ CJN.09180819

31 Vondran FWR, Timrott K, Kollrich S, Steinhoff AK, Kaltenborn A, Schrem H, Klempnauer J, Lehner F and Schwinzer R: Pretransplant immune state defined by serum markers and alloreactivity predicts acute rejection after living donor kidney transplantation. Clin Transplant 28: 968-979, 2014. PMID: 24931031. DOI: $10.1111 /$ ctr.12399

32 Redfield RR, Scalea JR, Zens TJ, Muth B, Kaufman DB, Djamali A, Astor BC and Mohamed M: Predictors and outcomes of delayed graft function after living-donor kidney transplantation. Transpl Int 29: 81-87, 2016. PMID: 26432507. DOI: $10.1111 /$ tri.12696

33 Azab B, Camacho-Rivera M and Taioli E: Average values and racial differences of neutrophil lymphocyte ratio among a nationally representative sample of United States subjects. PLoS One 9: 1-6, 2014. PMID: 25375150. DOI: 10.1371/journal. pone. 0112361

34 Forget P, Khalifa C, Defour JP, Latinne D, Van Pel MC and De Kock M: What is the normal value of the neutrophil-tolymphocyte ratio? BMC Res Notes 10: 1-4, 2017. PMID: 28057051. DOI: $10.1186 / \mathrm{s} 13104-016-2335-5$

Received August 28, 2020

Revised September 8, 2020 Accepted September 14, 2020 